BMJ Open Sport \& Exercise Medicine

\title{
Exercise for posterior tibial tendon dysfunction: a systematic review of randomised clinical trials and clinical guidelines
}

\author{
Megan H Ross, Michelle D Smith, Rebecca Mellor, Bill Vicenzino
}

\begin{abstract}
To cite: Ross MH, Smith MD, Mellor R, et al. Exercise for posterior tibial tendon dysfunction: a systematic review of randomised clinical trials and clinical guidelines. BMJ Open Sport \& Exercise Medicine 2018;4:e00430. doi:10.1136/ bmjsem-2018-000430
\end{abstract}

- Additional material is published online only. To view please visit the journal online (http://dx.doi.org/10.1136/ bmjsem-2018-000430).

Accepted 13 August 2018
Check for updates

(C) Author(s) (or their employer(s)) 2018. Re-use permitted under CC BY-NC. No commercial re-use. See rights and permissions. Published by BMJ.

Department of Physiotherapy, School of Health and Rehabilitation Sciences, The University of Queensland, Brisbane, Queensland, Australia

Correspondence to Professor Bill Vicenzino; b. vicenzino@uq.edu.au

\section{ABSTRACT}

Objective To systematically review all randomised clinical trials to determine the efficacy of local strengthening exercises compared with other forms of conservative management for adults with posterior tibial tendon dysfunction.

Design Systematic review.

Data sources Four electronic databases (Cumulative Index to Nursing and Allied Health Literature, Cochrane, Embase and PubMed) were searched up to June 2018. Eligibility criteria for selecting studies The study included randomised clinical trials investigating individuals with posterior tibial tendon dysfunction where local strengthening was compared with other forms of conservative management with respect to pain, function and/or physical impairment outcome measures. Standardised mean differences (SMDs) were used to compare change scores between groups and descriptors of exercise prescription assessed according to the Template for Intervention Description and Replication and the Toigo and Boutellier recommendations.

Results 3 studies $(n=93)$ were eligible for inclusion in the review. Varying strengthening exercises were compared with stretching and foot orthoses $(\mathrm{n}=2)$ or no intervention $(n=1)$. Moderate effects (SMD 0.6-1.2) were found for reducing pain and disability with eccentric strengthening in conjunction with stretching and orthoses compared with concentric exercises, stretching and orthoses combined, and stretching and orthoses alone. Evaluation of exercise prescription parameters demonstrated minimal reporting, with the only consistent parameters being the number of sets and repetitions of the exercises, and the duration of the experimental period.

Conclusion This review demonstrates the paucity of high-quality research for the conservative management of posterior tibial tendon dysfunction, and highlights the lack of exercise prescription parameters reported in clinical trials.

Trial registration number CRD42017076156.

\section{INTRODUCTION}

Posterior tibial tendon dysfunction (PTTD) is prevalent, with estimates of prevalence ranging between $3.3 \%$ and $10 \%,{ }^{1}$ but suspected to be much higher, as the condition
What is already known?

Posterior tibial tendon dysfunction is a progressive condition occurring along a continuum from tendon pain and dysfunction to acquired flatfoot deformity.

- Management in the early stages is typically conservative, focusing on local strengthening exercises and arch-supporting devices.

\section{What are the new findings?}

- High-quality clinical trials for the efficacy of exercise management in posterior tibial tendon dysfunction are lacking.

- Exercise prescription parameters are poorly reported in the literature.

- Preliminary evidence suggests exercise is beneficial in reducing pain and disability.

is often not formally diagnosed until the later stages. ${ }^{1}$ PTTD is progressive and disabling, characterised by impaired mobility, ${ }^{2}$ poor function, ${ }^{34}$ and often a range of comorbidities including hypertension and diabetes and higher body mass index (BMI). ${ }^{356}$

Decisions regarding management vary according to the stage of the pathology, ${ }^{7}$ with reports of surgery predominating, probably due to the condition more commonly presenting in later and more severe stages. Surgery aims to correct deformity in the later stages of the condition (ie, stages III and IV) ${ }^{9-11}$ and, relatively recently, to prevent soft tissue and joint destructions in earlier stages (I-II) that do not respond to conservative management. ${ }^{12-18}$

Conservative management is used in earlier stages (I-II) with a focus on local strengthening exercises for the tibialis posterior musculotendinous unit and use of an orthosis to brace the foot. ${ }^{19-21}$ The level of evidence in support of this approach is currently unevaluated and is the basis of this systematic review. 
In evaluating the level of evidence, it is important to also evaluate the quality of reporting of the exercise prescription parameters due to the potential influence variations in these parameters may have on the effectiveness of the treatment ${ }^{22}$ and on clinical practice.

The aims of this systematic review of randomised clinical trials (RCTs) were to provide estimates of treatment effects of local strengthening exercises compared with other forms of conservative management for adults with PTTD on outcomes relating to the international classification of functioning, disability and health (ICF) framework (impairments, activity limitations and participation restrictions), and to evaluate the completeness of exercise prescription descriptors.

\section{METHODS}

This systematic review was performed using a predetermined protocol in accordance with the Preferred Reporting Items for Systematic Reviews and Meta-Analyses statement. ${ }^{23}$ It was registered with PROSPERO (trial registration number CRD42017076156) and is available at http://www.crd.york.ac.uk/PROSPERO/display_ record.php?ID=CRD42017076156.

\section{Search strategy and data sources}

To answer the research question about the treatment effects of local strengthening exercises for PTTD, four electronic databases (Cumulative Index to Nursing and Allied Health Literature, Cochrane, Embase and PubMed) were searched from inception to June 2018 for full-text papers published in peer-reviewed journals. A comprehensive search strategy was developed to capture variations in terminology used in the literature for PTTD and key conservative interventions (online supplementary table 1). No further limits were applied to the initial search strategy. Reference list checks and author searches were also performed to ensure all relevant literature was identified.

\section{Eligibility criteria}

Eligibility criteria were determined prospectively using the patient, intervention, comparator and outcome (PICO) framework. ${ }^{24}$ Trials were eligible for inclusion if they investigated individuals with PTTD or adult-acquired flatfoot deformity due to PTTD, if they were randomised, and if local strengthening was compared with other forms of conservative management with respect to pain, function and/or physical impairment outcome measures. A diagnosis of PTTD was required to be made based on a minimal list of diagnostic criteria, ${ }^{25}$ with two or more of the following: tenderness on palpation of the posterior tibial tendon, pain and/or swelling along the posterior tibial tendon, medial foot pain, difficulty and/or pain with single leg heel raise, and inability to invert the calcaneus on double leg heel raise. Flatfoot deformity was not considered as a selection criterion and as such all stages of PTTD were included.
Trials were excluded if they compared surgical interventions for PTTD, did not include a comparator group and combined data for individuals diagnosed with conditions other than PTTD. Reviews, case studies and trials for paediatric flatfoot, asymptomatic flatfoot, neurological conditions and rheumatoid arthritis were also excluded.

\section{Study selection}

The lead reviewer (MHR) performed the search and exported all retrieved records into EndNote V.X7 (Thompson Reuters, Carlsbad, California, USA). Duplicates were removed and titles and abstracts were screened independently by two reviewers (MHR and RM), based on established eligibility criteria. Full texts were retrieved for all potentially eligible papers and reviewed for inclusion and exclusion criteria. Where there were uncertainties, at least one additional author (MDS or BV) was consulted to determine final eligibility.

\section{Data extraction}

Data extraction for each included trial was completed by two investigators (MHR and RM) using a predetermined spreadsheet. Where reference was made to protocol papers or supplementary materials, these sources were obtained and used for data extraction. For each trial, study design, sample size, participant characteristics/ demographics, diagnostic criteria, methods, intervention details (type, frequency, duration), outcomes, follow-up and results (means and SDs) for each time point were extracted.

As reporting of parameters of exercise prescription is essential for the implementation of research findings in exercise therapy, these data were also extracted. The 'Template for Intervention Description and Replication' (TIDieR) checklist ${ }^{26}$ (developed to facilitate reporting and replication of intervention studies) and the guidelines developed by Toigo and Boutellier ${ }^{27}$ specifically for resistance exercise prescription provide a framework appropriate for the appraisal of exercise prescription in intervention studies for musculoskeletal conditions. ${ }^{28} \mathrm{As}$ such, specific parameters (\% repetition maximum, repetitions, time under tension and so on) were extracted to allow for analysis of mechanobiological descriptors of exercise prescription. ${ }^{27}$ Data for the 12 -item TIDieR checklist $^{26}$ were also independently extracted by two reviewers, and the completeness of reporting was evaluated by allocating 1 point for complete items (clear, unambiguous descriptions allowing replication) and 0 for incomplete items (partial or no description) as per Holden et al..$^{28}$ The total scores were calculated for each checklist and two authors (MDS and BV) verified all extracted data for accuracy.

\section{Risk of bias}

Risk of bias was assessed as recommended by The Cochrane Collaboration's tool for assessing risk of bias in randomised trials. ${ }^{24}$ The tool assesses six potential sources of bias under five domains (selection bias, 
performance bias, detection bias, attrition bias and reporting bias) and considers each as being either 'low risk', 'high risk' or 'unclear risk' of bias. Two independent reviewers (MHR and RM) rated included trials and the results were collated and examined for discrepancies. Inter-rater disagreements were discussed and where a consensus could not be met were taken to a third party (BV or MDS).

\section{Statistical analyses/data synthesis}

Analyses were performed in Review Manager (RevMan) V.5.3 (Copenhagen: The Nordic Cochrane Centre, The Cochrane Collaboration). For continuous measures of pain, function and/or physical impairment, individual study effect sizes were expressed as standardised mean differences (SMDs) using means and SDs. The mean change scores from preintervention to postintervention were compared between two independent participant groups (ie, strengthening vs no strengthening; type of strengthening comparison). Change scores (mean and SD) for each group were calculated as postscore minus prescore with within-group correlation assumed to be $0.5^{24}$ and were used to estimate the SD of the mean change using t-distributions.

The difference between each group was considered significant where $95 \%$ CIs did not contain 0 . For pain and self-reported outcome measures, higher scores indicated worse outcomes, and as such the inverse of effect size was reported so that positive effect sizes indicated a beneficial effect for the intervention group. Improvements in strength and function measures were indicated by higher scores and positive effect sizes. The strength of the effect size was interpreted based on Hopkins, ${ }^{29}$ as follows: $<0.2$ trivial effect, 0.2-0.6 small effect, 0.61-1.2 medium effect, $>1.2-2.0$ large effect and 2.0-4.0 extremely large effect.

Inter-rater reliability of methodological quality was calculated in Stata V.13 using the K-statistic (95\% CI). The reliability of the quality ratings between the two assessors was interpreted as $\mathrm{K}<0.00$ poor agreement, $0.00-0.20$ slight agreement, $0.21-0.40$ fair agreement, $0.41-0.60$ moderate agreement, $0.61-0.80$ substantial agreement and $0.81-1.00$ almost perfect agreement. ${ }^{30}$

\section{RESULTS}

\section{Study selection and design}

The electronic database search retrieved 347 studies. After removing duplicates, 242 titles and abstracts were screened and 16 potentially eligible full-text trials were assessed for eligibility (figure 1). Three randomised controlled trials were included in qualitative and quantitative synthesis.

\section{Risk of bias}

The inter-rater reliability for the risk of bias assessment was almost perfect (agreement on 16 of 18 ratings, $\mathrm{K}=0.857(0.47-1))$. Risk of bias was variable across the six items, with insufficient information available to permit a judgement for two of the three trials on four items (random sequence generation, allocation concealment, blinding of participants and personnel, blinding

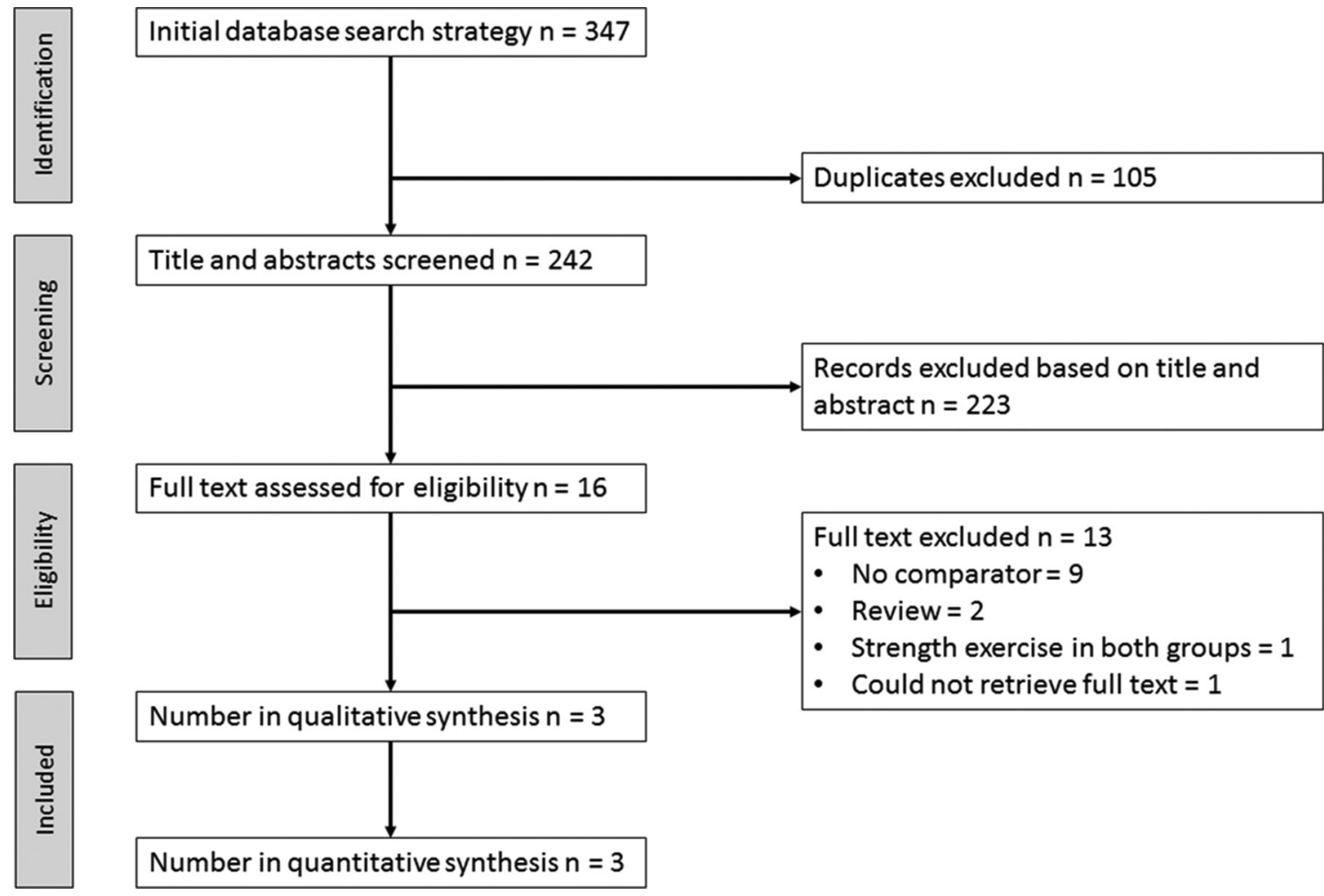

Figure 1 Preferred Reporting Items for Systematic Reviews and Meta-Analyses flow chart for selected trials included for the systematic review. 
of outcome assessment; online supplementary table 2). Considering attrition, two trials were deemed to have low risk of bias as reasons were provided for missing data (dropouts), which were unrelated to outcomes of the intervention, and dropouts were balanced across groups. The third trial had an imbalance of missing data across groups (2 (29\%) vs $0(0 \%))$ for all outcomes, and due to the already small sample size $(n=14)$ it is plausible this was large enough to induce clinically relevant bias. Selective reporting overall had a high risk of bias. Of the two trials in which a judgement could be made, there were outcomes specified in the trial protocol that were omitted from the final analyses and the manuscript.

\section{Participant characteristics}

A total of 93 individuals with PTTD were enrolled across all trials, with individual sample sizes ranging from 14 to 40 participants (5-19 per group) (table 1). Studies enrolled participants with a mean age from $52.9^{31}$ to $57.5^{32}$ years and BMI between $23.3^{31}$ and $30.5^{32} \mathrm{~kg} / \mathrm{m}^{2}$. All studies had a predominance of women, with the percentage of women ranging from $77.7 \%{ }^{32} 33$ to $100 \% .^{31}$ Two trials ${ }^{3133}$ included individuals with stage I or II PTTD, and one trial $^{32}$ included those with only stage II PTTD.

\section{Selection criteria}

In all trials, diagnosis of PTTD was established based on physical examination findings performed by either physical therapists or foot and ankle physicians. The number of essential/compulsory diagnostic criteria ranged between two and six, with pain along the posterior tibial tendon, tenderness on palpation of the posterior tibial tendon, medial foot pain and a correctable flatfoot deformity most frequently used (table 2). Imaging was not used in any trial to confirm diagnosis or exclude other potential sources of pain. Only one trial ${ }^{33}$ reported a minimum duration of symptoms and one reported restrictions in function (able to walk $>15 \mathrm{~m}$ ) and age ( $>40$ years) ${ }^{32}$ as study selection criteria.

\section{Outcome measures}

The trials included in this review reported a range of outcome measures relating to physical impairment, pain and function (online supplementary table 3). Two studies ${ }^{31} 33$ used a Visual Analogue Scale (VAS) to measure pain immediately post 5 min walk test (5MWT). The same two studies ${ }^{31} 33$ also reported distance ambulated $(\mathrm{m})$ during the 5MWT. Houck et $a l^{32}$ reported tibialis posterior muscle torque with combined plantarflexion and inversion, whereas Jeong $e t a l^{11}$ reported ankle strength and range of motion in dorsiflexion, plantarflexion, inversion and eversion. A total of three patient-reported outcome measures were used, with two trials $^{32}{ }^{33}$ reporting the Foot Function Index (FFI). The FFI consists of three domains (pain, disability and activity limitations) which are summed to provide an overall total score. Houck et $a l^{32}$ also used the Short Musculoskeletal Function Assessment (SMFA), which consists of mobility,

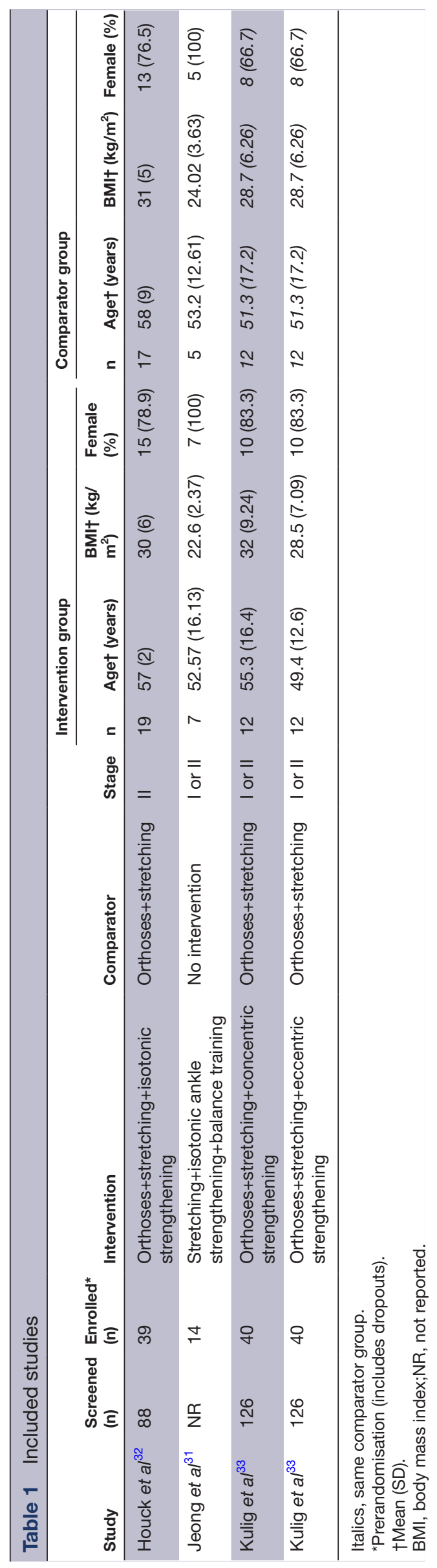


Table 2 Selection criteria as stated in each study

\begin{tabular}{|c|c|c|c|c|c|c|c|c|c|c|}
\hline Trial & $\begin{array}{l}\text { Medial } \\
\text { foot/ankle } \\
\text { pain }\end{array}$ & $\begin{array}{l}\text { Pain } \\
\text { PTT }\end{array}$ & $\begin{array}{l}\text { Swelling } \\
\text { of PTT }\end{array}$ & $\begin{array}{l}\text { TOP } \\
\text { PTT }\end{array}$ & $\begin{array}{l}\text { Correctable } \\
\text { flatfoot } \\
\text { deformity }\end{array}$ & $\begin{array}{l}\text { Foot } \\
\text { flattening }\end{array}$ & $\begin{array}{l}\text { Abducted } \\
\text { mid-foot }\end{array}$ & $\begin{array}{l}\text { Duration of } \\
\text { symptoms }\end{array}$ & Imaging & Other inclusion criteria \\
\hline Houck et $a l^{32}$ & NR & Either & & NR & $\sqrt{ }$ & NR & NR & NR & NR & $\begin{array}{l}\text { Able to walk }>15 m \\
>40 \text { years of age }\end{array}$ \\
\hline Jeong et $a l^{31}$ & $\sqrt{ }$ & $\sqrt{ }$ & NR & $\sqrt{ }$ & NR & NR & NR & NR & NR & NR \\
\hline Kulig et $a l^{33}$ & $\sqrt{ }$ & NR & NR & $\sqrt{ }$ & $\sqrt{ }$ & $\sqrt{ }$ & $\sqrt{ }$ & $>3$ months & NR & NR \\
\hline
\end{tabular}

$\sqrt{ }$, essential eligibility criteria for the study; either, one finding from this group of tests/clinical findings was required.

NR, not reported; PTT, posterior tibial tendon; TOP, tender on palpation.

dysfunction and bother indexes. Jeong et $a l^{31}$ reported the American Orthopaedic Foot and Ankle Society score, which combines both patient self-report and clinician physical examination findings into one aggregate score. ${ }^{34}$ Reassessment of outcomes varied from 6 weeks ${ }^{31}{ }^{32}$ to 12 weeks. $^{32} 33$

\section{Interventions}

The exercise intervention protocol varied in each of the included trials. Local tibialis posterior exercises were compared with foot orthoses and stretching in two trials $^{32}{ }^{33}$; however, the type of exercise (concentric, eccentric or isotonic) varied. Participants in the Kulig $e t a l^{33}$ trial were randomly assigned to either an eccentric or concentric exercise group (combined with stretching and orthoses) or a stretching and orthoses-only group (three groups in total). Houck et $a l^{32}$ used an isotonic strengthening regimen combined with stretching and orthoses compared with stretching and orthoses only. Participants in the Jeong et $a \hat{l}^{31}$ trial were randomised to receive either an isotonic ankle strengthening, stretching and balance programme, or no intervention.

\section{Completeness of reporting}

Completeness of intervention reporting based on the TIDieR checklist is provided in online supplementary table 4. Of the 12 items, Jeong $e t a l^{\beta 1}$ provided adequate information for 4 items, Houck $e t a l^{2}$ for 11 items and Kulig $e t a \vec{l}^{33}$ for all 12 items. Houck $e t a \vec{l}^{2}$ and Kulig $e$ t $a \vec{l}^{33}$ both included sufficient information in regard to adherence (both the plan for assessment of adherence and reports of actual adherence).

No trial provided complete reporting of interventions based on the Toigo and Boutellier ${ }^{27}$ exercise prescription descriptors (table 3). Of the 13 items, Jeong et $a l^{31}$ provided adequate information for 5 items, Houck $e t a l^{32}$ for 7 items and Kulig $e t a l^{33}$ for 11 items. Of the six classical descriptors, only the number of sets and repetitions of the exercises and duration of the experimental period over which exercises were performed were consistently described for all exercises in all trials (table 3). Load magnitude (\% repetition maximum) was only described in one trial. ${ }^{33}$ Of the seven remaining mechanobiological descriptors, the range of motion and an anatomical definition of the exercise were described in the methodology of two trials, ${ }^{32}{ }^{33}$ and time under tension was described in one trial. ${ }^{33}$

\section{Main findings}

Physical impairments

Isotonic ankle strengthening, balance and stretching improved ankle dorsiflexion range at 6 weeks beyond that of no intervention (SMD (95\% CI) 1.71 (0.29 to 3.12)) (figure 2). Plantar flexion inversion torque was not different at 6 weeks following isotonic tibialis posterior strengthening exercise combined with stretching and orthoses compared with stretching and orthoses alone (SMD (95\% CI) 0.59 (-0.08 to 1.26) ) (figure 2). Isotonic ankle strengthening, balance and stretching did not improve ankle torque in any direction at 6 weeks beyond that of no intervention (figure 2). Local strengthening was not superior to control comparator for any other physical impairment outcomes at 6 weeks (figure 2 ).

Neither concentric nor eccentric tibialis posterior strengthening exercises combined with stretching and orthoses were significantly different from the control for the distance covered during the 5MWT at 12 weeks (SMD (95\% CI) 0.51 ( -0.34 to 1.36$)$ and 0.25 ( -0.57 to 1.07 ), respectively), ${ }^{33}$ nor were there differences between eccentric and concentric strengthening groups (SMD (95\% CI) $-0.39(-1.22$ to 0.44$)$ ) (figure 3$)$. There was no difference between isotonic tibialis posterior strengthening and the control group for tibialis posterior strength (isometric combined plantarflexion and inversion) at 12 weeks (SMD (95\% CI) 0.59 (-0.08 to 1.26)) (figure 3$){ }^{32}$

\section{Patient-reported outcomes}

Isotonic ankle strengthening, balance and stretching reduced pain on VAS beyond that of no intervention, with a large, significant effect size at 6 weeks (SMD (95\% CI) -2.39 (-4.02 to -0.75$)$ ) (figure 2$).{ }^{31}$ Isotonic strengthening moderately reduced scores for the mobility and dysfunction subscales of the SMFA at 6 weeks (SMD $(95 \%$ CI) $-1.10(-1.81$ to -0.4$)$ and $-0.87(-1.55$ to -0.18$)$, respectively) (figure 2), but not at 12 weeks (SMD $(95 \%$ CI) 0.32 ( -0.98 to 0.34$)$ and -0.41 ( -1.07 to 0.26$)$, respectively) (figure 3)..$^{32}$ There were no differences between local strengthening and control groups for the mean change on the FFI subscales or total score at 6 weeks (figure 2) or the SMFA bother subscale at 6 or 12 weeks (figure 3). ${ }^{32}$

Eccentric strengthening combined with stretching and orthoses reduced the mean scores for FFI-pain, 


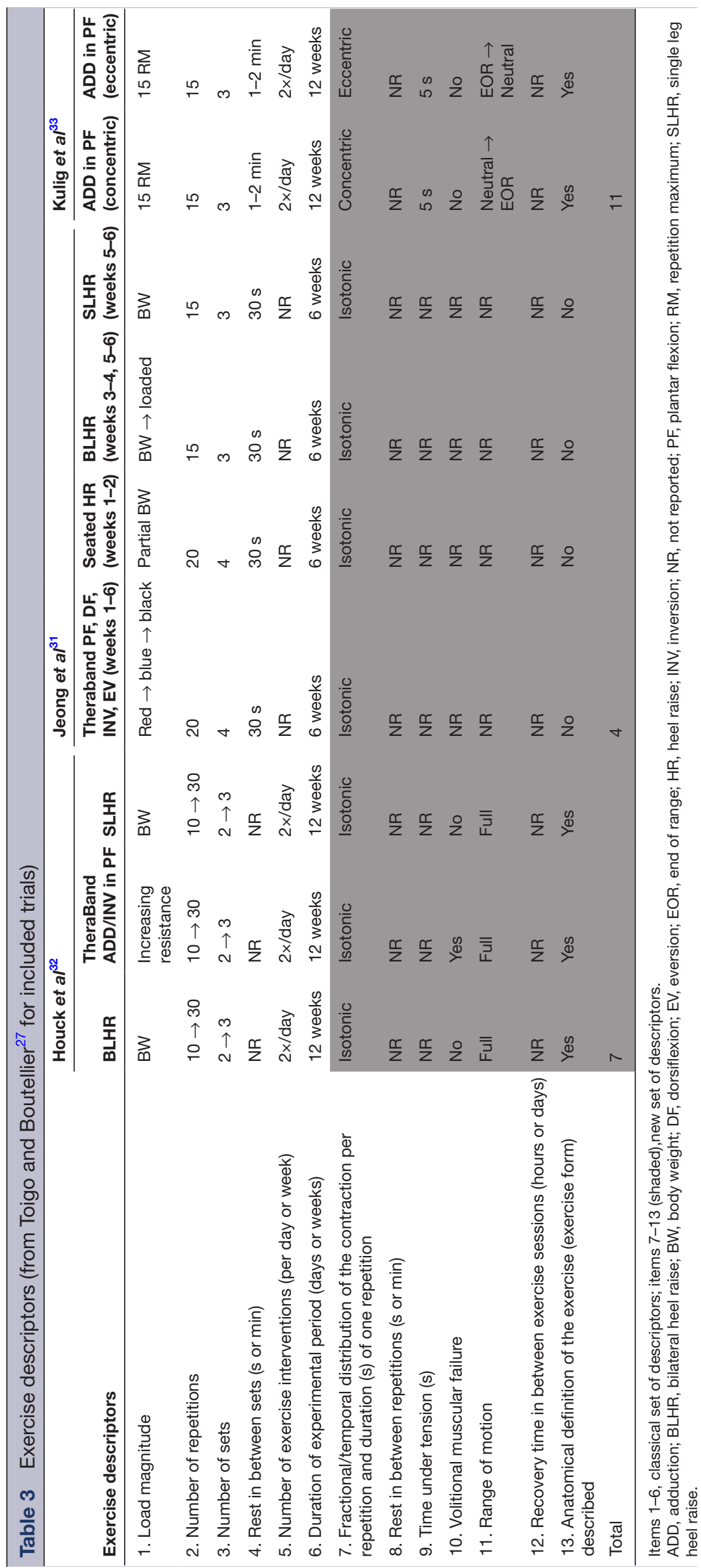




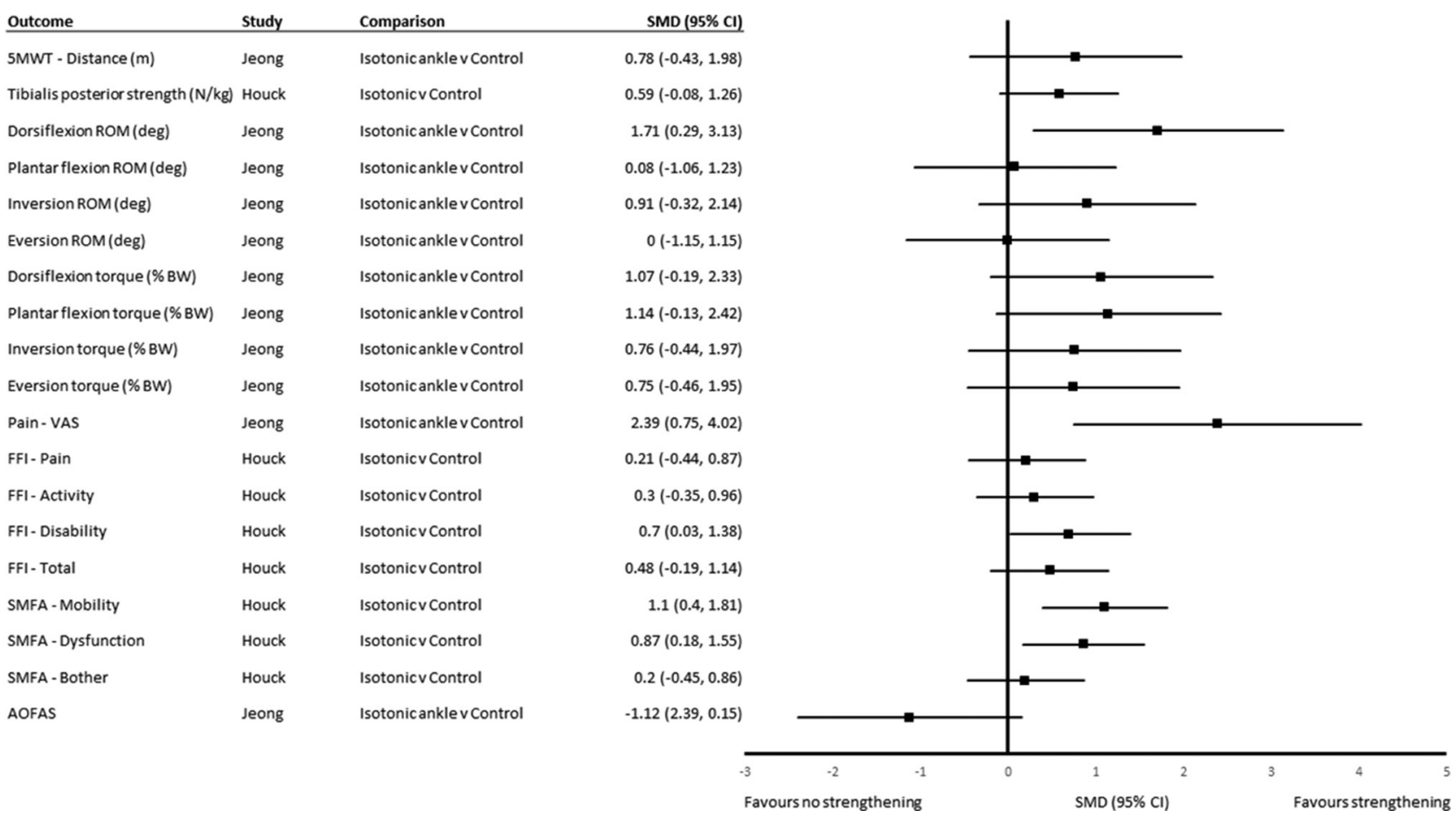

Figure 2 SMD $(95 \% \mathrm{Cl})$ for outcomes at 6 weeks. 5MWT, 5 min walk test; AOFAS, American Orthopaedic Foot and Ankle Society; BW, body weight; deg, degree; FFI, Foot Function Index; N/kg, Newtons per kilogram; ROM, range of motion; SMD, standardised mean difference; SMFA, Short Musculoskeletal Function Assessment; VAS, Visual Analogue Scale.

FFI-disability and FFI-total beyond that of concentric strengthening, stretching and orthoses combined, and stretching and orthoses alone at 12 weeks with moderate effect sizes (SMD (95\% CI) $-1.1(-1.97$ to -0.23$),-0.97$ $(-1.82$ to -0.11$)$ and -0.96 (1.81 to -0.1$)$, respectively; and SMD (95\% CI) $1.1(-1.97$ to -0.23$),-0.96(-1.81$ to -0.11$)$ and $-0.85(-1.69$ to -0.01$)$, respectively) (figure 3$).{ }^{33}$ Neither concentric nor isotonic tibialis posterior strengthening combined with stretching and orthoses was significantly different from stretching and orthoses alone for the three subscales of the FFI and FFI-total at 12 weeks (figure 3). ${ }^{32} 33$

\begin{tabular}{|c|c|c|c|}
\hline Outcome & Study & Comparison & SMD $(95 \%$ a) \\
\hline \multirow[t]{3}{*}{ 5MWT - Distance (m) } & Kulig & ConcentricvControl & $0.51(-0.34,1.36)$ \\
\hline & Kulig & Eccentric vControl & $0.25(-0.57,1.07)$ \\
\hline & Kulig & EccentricvConcentric & $-0.39(-1.22,0.44)$ \\
\hline Tibialis poster ior strength $(\mathrm{N} / \mathrm{kg})$ & Houck & Isotonicv Control & $0.59(-0.08,1.26)$ \\
\hline \multirow[t]{3}{*}{ Pain-VAS } & Kulig & ConcentricvControl & $0.54(-0.32,1.39)$ \\
\hline & Kulig & EccentricvControl & $0.56(-0.28,1.39)$ \\
\hline & Kulig & EccentricvConcentric & $0.24(-0.58,1.06)$ \\
\hline \multirow[t]{4}{*}{ FFI-Pain } & Houck & Isotonicvcontrol & $0.12(-0.54,0.77)$ \\
\hline & Kulig & ConcentricvControl & $0.28(-0.52,1.09)$ \\
\hline & Kulig & Eccentricv Control & $1.1(0.23,1.97)$ \\
\hline & Kulig & Eccentricv Concentric & $0.88(0.03,1.72)$ \\
\hline \multirow[t]{4}{*}{ FFI-Activity } & Houck & Isotonicv Control & $0(-0.65,0.65)$ \\
\hline & Kulig & ConcentricvControl & $-0.21(-1.01,0.59)$ \\
\hline & Kulig & Eccentricvcontrol & $0.07(-0.73,0.87)$ \\
\hline & Kulig & Eccentricv Concentric & $0.39(-0.41,1.2)$ \\
\hline \multirow[t]{4}{*}{ FFI-Disability } & Houck & IsotonicvControl & $0.19(-0.47,0.84)$ \\
\hline & Kulig & ConcentricvControl & $0.14(-0.67,0.94)$ \\
\hline & Kulig & Eccentric vControl & $0.96(0.11,1.81)$ \\
\hline & Kulig & EccentricvConcentric & $0.97(0.11,1.82)$ \\
\hline \multirow[t]{4}{*}{ FFI-Total } & Houck & Isotonicycontrol & $0.08(-0.58,0.73)$ \\
\hline & Kulig & Concentricv Control & $0.09(-0.71,0.89)$ \\
\hline & Kulig & Eccentricv Control & $0.85(0.01,1.69)$ \\
\hline & Kulig & Eccentricv Concentric & $0.96(0.1,1.81)$ \\
\hline SMFA-Mobility & Houck & Isotonic vControl & $0.32(-0.34,0.98)$ \\
\hline SMFA-Dysfunction & Houck & IsotonicvControl & $0.41(-0.26,1.07)$ \\
\hline SMFA-Bother & Houck & IsotonicvControl & $-0.17(-0.82,0.49)$ \\
\hline
\end{tabular}

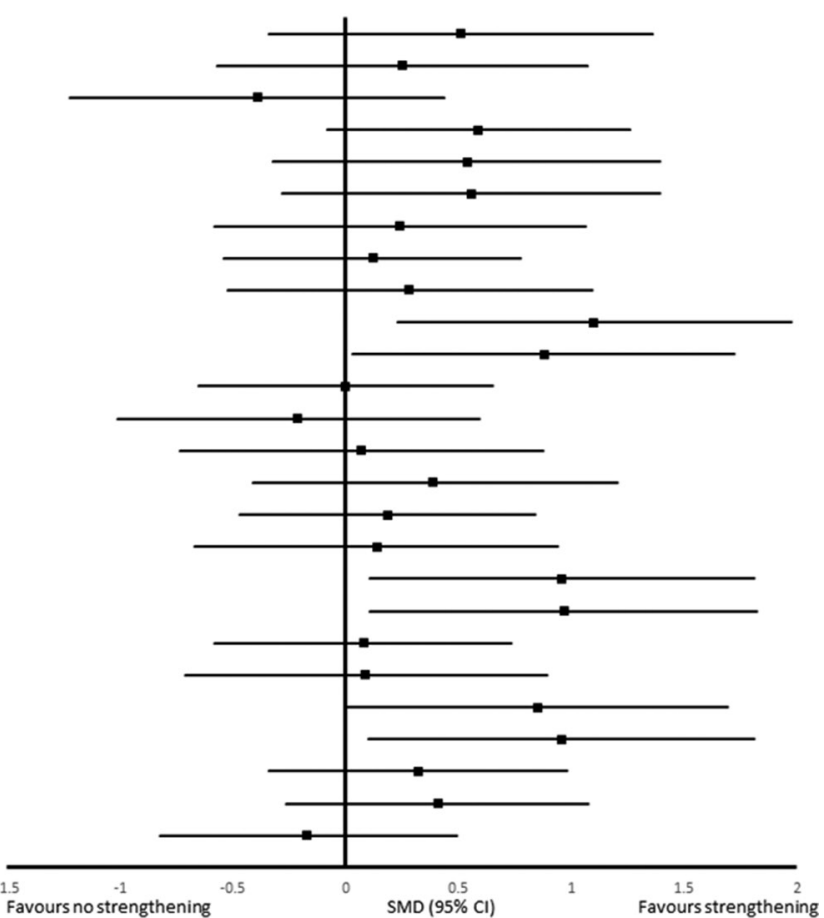

Figure $3 \mathrm{SMD}(95 \% \mathrm{Cl})$ for outcomes at 12 weeks. 5MWT, 5 min walk test; FFI, Foot Function Index; SMD, standardised mean differences; SMFA, Short Musculoskeletal Function Assessment; VAS, Visual Analogue Scale. 


\section{DISCUSSION}

This systematic review evaluated pain and functional outcomes following local strengthening exercise in individuals with PTTD. Two main findings emanate from this systematic review: the first is the lack of rigorous RCTs investigating the effects of non-surgical management on impairments, activity limitations and participation in adults with PTTD, and the second is that exercise parameters are poorly reported.

Detailed reporting of exercise parameters for musculoskeletal interventions trialled in RCTs is essential for clinical replication and translation of research into practice. The implications of omitting important exercise parameters in reporting, however, extend beyond just clinical replication of exercise prescription. Exercise parameters such as time under tension, range of motion and rest or recovery time can be manipulated and are expected to influence both physiological response to and efficacy of the exercise prescription, ${ }^{22} 27$ meaning that slight variations in prescription parameters may have vastly different physiological effects. Factors related to biophysical response to exercise were not sufficiently described in the included studies, and strengthening interventions failed to improve strength-related outcome measures at both 6 and 12 weeks. Lack of detailed reporting becomes an important matter when a primary goal in rehabilitation of tendinopathies is to improve the load management capacity of the musculotendinous unit. $^{35}$

Current literature implicates appropriate load management as the most important component of rehabilitation for tendinopathies. ${ }^{35-37}$ The benefit of therapeutic exercise in the management of lateral epicondylalgia and Achilles, patellar and rotator cuff tendinopathies has been established in previous systematic reviews. ${ }^{38-41}$ While early literature has focused on eccentric exercise for tendinopathies, ${ }^{42-44}$ more recent approaches with good efficacy include patient education on load management strategies and individualised, progressive loading exercises. ${ }^{45}$ Overall, effect sizes from this systematic review provide limited evidence to suggest that isotonic tibialis posterior strengthening, stretching and orthoses and general isotonic ankle strengthening, balance and stretching exercises similarly improve pain, mobility and dysfunction in PTTD in the short term compared with no strengthening. Considering the specific type of strengthening protocol, data from this review suggest that eccentric strengthening may be marginally more effective than other types of strengthening, with eccentric but not concentric exercise resulting in significant reductions in self-reported pain, disability and overall foot function compared with controls at 12 weeks.

The mechanism of effect for improved outcomes in tendinopathy following strengthening exercise is understood to be related to load. It has been suggested that the load through the tendon during therapeutic exercises needs to be sufficiently high enough to elicit physiological changes within the tendon. While the relationships between internal tendon structure and pain and function are currently unclear, ${ }^{37}$ heavy-slow resistance appears to be beneficial in managing Achilles and patellar tendinopathies. ${ }^{46}$ It has been suggested that the physiological response to therapeutic exercise may be greater with heavy-slow resistance and eccentric strengthening due to higher loads applied through the tendon during these exercises. The device used for strengthening exercise in the study by Kulig $e t a l^{33}$ allowed for quantification of load and constant resistance throughout the exercise. Participants in the eccentric exercise group in their clinical trial achieved loads 3.3 times higher than those in the concentric group by the end of the 12-week intervention. ${ }^{33}$ This raised the possibility that differences in outcomes were dependent on load rather than specific contraction type. Tolerance and ability to perform the exercise with good form were the criteria for progressing load, which suggests that participants in the eccentric group were better able to tolerate higher loads during the exercise programme, optimising tendon response, and leading to the reporting of greater improvements in pain, disability and overall foot function. Physical tests of function (distance covered during 5MWT), however, were not different between groups. This suggests that while participants felt more confident loading their tendon, physical capacity of the tendon might not have improved.

Exercise prescription parameters can be manipulated depending on the desired physiological response to exercise stimulus, for example, to improve skeletal muscle strength, endurance or power. Each of the three trials indicated that the intention of the prescribed exercises was to improve strength. On further examination of the exercise prescription parameters (table 3 ) in reference to the current American College of Sports Medicine (ACSM) guidelines for muscular strength, ${ }^{47}$ some discrepancies were apparent. Considering load magnitude, the ACSM guidelines for strength recommend up to 12 repetition maximum, where Kulig et $a l^{33}$ prescribed 15, fitting the ACSM guidelines for muscular endurance. ${ }^{47}$ Similarly, papers prescribed between 15 and 30 repetitions, which is above the recommendations for inducing strength adaptations (8-12) and falls into the recommended repetitions for improving muscular endurance. ${ }^{47}$

Adherence should be considered in calculating the exercise stimulus (load) actually delivered to the musculotendinous unit and any strength gains accrued. Adherence was not reported in Jeong et al, ${ }^{31}$ but ranged between $29 \%$ and $126 \%$ (average $79 \%$ ) in Houck et $a l^{32}$ and $39 \%-98 \%$ (average $=68 \%$ ) in Kulig et al. ${ }^{33}$ Considering this, it is possible the actual load participants performed was not high enough to elicit adaptations in skeletal muscle that would subsequently result in clinical improvements in strength (Houck et $a l^{2}$ ) or physical tests of function (Kulig et $a \vec{l}^{33}$ ). Houck et $a l^{32}$ examined tibialis posterior force production in plantarflexion and forefoot adduction at baseline and at 6 and 12 weeks following isotonic tibialis posterior exercises against the heaviest TheraBand resistance that could be tolerated, 
in addition to bilateral and unilateral heel raises. ${ }^{32}$ The strengthening group did not exhibit increases in tibialis posterior strength at 6 or 12 weeks, which suggests that while the intention of the prescribed exercise programme was to increase strength, with poor adherence taken into consideration, actual load may not have been appropriate to elicit changes in musculotendinous strength.

It was common among included trials for the intervention protocol to include cointerventions such as stretching and orthoses, in addition to specific local strengthening exercises. It is possible that the effect of the local strengthening intervention was affected by these cointerventions. As no randomised trial has looked at local strengthening in isolation (ie, not combined with stretching/orthoses or balance and stretching exercises), it is difficult to ascertain to what degree improvements can be attributed to targeted exercises only. Two trials that investigated stretching, orthoses and local strengthening compared with stretching and orthoses alone showed similar improvement in pain and function in all groups. It is possible that orthoses and/or stretching play a role in the reduction of pain. Future research is required to investigate strength interventions in isolation of other treatments to establish its efficacy in the management of PTTD.

Interestingly, stretching exercises were included in all intervention groups across the three included trials. Both gastrocnemius and soleus stretches were prescribed for 3-10 repetitions of $30 \mathrm{~s}$ duration, 2-4 times per day. This stretch is performed in maximal dorsiflexion, which increases the compressive as well as the tensile load on the posterior tibial tendon posterior to the medial malleolus, ${ }^{48}$ the combination of which has been found to be most damaging to the tendon. ${ }^{49}$ Load management for pain relief in tendinopathy rehabilitation is twofold, incorporating the reduction of both compressive and tensile loads. ${ }^{50}$ So while foot orthoses and activity modification may aid in altering tensile loads (supporting the medial longitudinal arch and reducing the torque required from the tibialis posterior during activities), accompanying these interventions with static stretches in full dorsiflexion may be counterproductive to pain management and rehabilitation.

Pain with palpation, pain on tendon loading and impaired function are key features in the clinical presentation of tendinopathies. ${ }^{51-54}$ Pain and difficulty during activities that load the medial aspect of the foot and the posterior tibial tendon, such as the single leg heel raise, are key clinical features of PTTD. The results from this systematic review have highlighted that interventions that aim to modify the load through the tendon and foot locally (ie, via tibialis posterior strengthening and/ or arch-supporting devices such as foot orthoses) have limited ability to improve pain and functional outcomes in PTTD. As such, alternative means of modifying load to improve clinical outcomes warrant further investigation. Hip function can affect motion at the foot during gait, ${ }^{55-57}$ and weak hip external rotators and abductors have been associated with increased femoral internal rotation $^{5859}$ and adduction, ${ }^{60}$ increased knee valgus, ${ }^{5961}$ tibial internal rotation ${ }^{61-63}$ and subtalar joint pronation, ${ }^{6264}$ which may impact on tibialis posterior. Increased rearfoot eversion ${ }^{65-69}$ and hip abduction strength ${ }^{2}$ deficits have been demonstrated in PTTD, which suggests that some proximal changes may be evident in the condition. Further research investigating proximal muscle function and kinematics in PTTD would provide further support for interventions targeting proximal hip motor control and strength.

\section{Limitations}

While this is the first systematic review to investigate the efficacy of exercise as a treatment for PTTD, there are several limitations that must be acknowledged. The small number and variability of interventions and outcomes of included studies did not allow meta-analysis or pooling of results. Meta-analysis was prevented due to variability in selection criteria, methodological quality, interventions and outcome measures assessed among the three included studies. Small sample sizes of individual studies can influence the ability to detect true effects. With very few outcomes replicated between studies, meta-analysis was prohibited and effect sizes presented in this review should be interpreted with this in mind. These aspects of the literature limit the inferences that might be drawn from the findings. Notwithstanding, this review is a synthesis of all available evidence from randomised controlled trials relating to exercise management for PTTD and highlighted the dearth of evidence on which to guide management. It must be acknowledged that studies included in this review related to stage I and/or II PTTD only. This is an important consideration in terms of the clinical application of findings and the generalisability of results, given that patient presentation may vary as the condition progresses.

\section{CONCLUSION}

This is the first systematic review on exercise therapy for PTTD. Based on the limited available literature, it appears that local strengthening exercises provide some benefit in PTTD, and eccentric exercises may be superior for improving pain, disability and self-reported overall foot function than concentric exercises and foot orthoses and stretching alone. No recommendations can currently be made regarding optimal exercise prescription based on published clinical trials. Clinicians should be guided by presenting impairments to prescribe exercise, which holds some promise in managing PTTD.

Contributors All authors contributed equally to this manuscript.

Funding BV is supported by the National Health and Medical Research Council (NHMRC) Program Grant (\#631717), MHR is supported by the University of Queensland Research Training Program (RTP) Scholarship

Competing interests None declared.

Patient consent Not required.

Provenance and peer review Not commissioned; internally peer reviewed. 
Open access This is an open access article distributed in accordance with the Creative Commons Attribution Non Commercial (CC BY-NC 4.0) license, which permits others to distribute, remix, adapt, build upon this work non-commercially, and license their derivative works on different terms, provided the original work is properly cited, appropriate credit is given, any changes made indicated, and the use is non-commercial. See: http://creativecommons.org/licenses/by-nc/4.0/

\section{REFERENCES}

1. Kohls-Gatzoulis J, Woods B, Angel JC, et al. The prevalence of symptomatic posterior tibialis tendon dysfunction in women over the age of 40 in England. Foot Ankle Surg 2009;15:75-81.

2. Kulig K, Popovich JM, Noceti-Dewit LM, et al. Women with posterior tibial tendon dysfunction have diminished ankle and hip muscle performance. J Orthop Sports Phys Ther 2011;41:687-94.

3. Chimenti RL, Tome J, Hillin CD, et al. Adult-acquired flatfoot deformity and age-related differences in foot and ankle kinematics during the single-limb heel-rise test. J Orthop Sports Phys Ther 2014:44:283-90.

4. Neville C, Flemister A, Tome J, et al. Comparison of changes in posterior tibialis muscle length between subjects with posterior tibial tendon dysfunction and healthy controls during walking. J Orthop Sports Phys Ther 2007;37:661-9.

5. Holmes GB, Mann RA. Possible epidemiological factors associated with rupture of the posterior tibial tendon. Foot Ankle 1992;13:70-9.

6. Rosenberg ZS, Jahss MH, Noto AM, et al. Rupture of the posterior tibial tendon: CT and surgical findings. Radiology 1988;167:489-93.

7. Johnson KA, Strom DE. Tibialis posterior tendon dysfunction. Clin Orthop Relat Res 1989;239:196-206.

8. Kohls-Gatzoulis J, Angel J, Singh D. Tibialis posterior dysfunction as a cause of flatfeet in elderly patients. Foot 2004;14:207-9.

9. Lombardi CM, Dennis LN, Connolly FG, et al. Talonavicular joint arthrodesis and evans calcaneal osteotomy for treatment of posterior tibial tendon dysfunction. J Foot Ankle Surg 1999;38:116-22.

10. Myerson MS, Corrigan J, Thompson F, et al. Tendon transfer combined with calcaneal osteotomy for treatment of posterior tibial tendon insufficiency: a radiological investigation. Foot Ankle Int 1995;16:712-8.

11. Mehta SK, Kellum RB, Robertson GH, et al. Radiographic correction of stage III posterior tibial tendon dysfunction with a modified triple arthrodesis. Foot Ankle Int 2013;34:1355-63.

12. Conti MS, Chan JY, Do HT, et al. Correlation of postoperative midfoot position with outcome following reconstruction of the stage II adult acquired flatfoot deformity. Foot Ankle Int 2015;36:239-47.

13. Conti MS, Ellis SJ, Chan JY, et al. Optimal position of the heel following reconstruction of the stage II adult-acquired flatfoot deformity. Foot Ankle Int 2015;36:919-27.

14. Soukup DS, MacMahon A, Burket JC, et al. Effect of obesity on clinical and radiographic outcomes following reconstruction of stage II adult acquired flatfoot deformity. Foot Ankle Int 2016;37:245-54.

15. Zhu Y, Xu XY. Treatment of stage II adult acquired flatfoot deformity with subtalar arthroereises. Foot Ankle Spec 2015;8:194-202.

16. Teasdall RD, Johnson KA. Surgical treatment of stage I posterior tibial tendon dysfunction. Foot Ankle Int 1994;15:646-8.

17. Crates JM, Richardson EG. Treatment of stage I posterior tibial tendon dysfunction with medial soft tissue procedures. Clin Orthop Relat Res 1999;365:46-9.

18. Sharma $P$, Singh SK, Rao SG. Is there a role for surgical decompression in stage I tibialis posterior tendon dysfunction? Foot 2003;13:1-4

19. Kulig K, Lederhaus ES, Reischl S, et al. Effect of eccentric exercise program for early tibialis posterior tendinopathy. Foot Ankle Int 2009;30:877-85.

20. Alvarez RG, Marini A, Schmitt C, et al. Stage I and II posterior tibial tendon dysfunction treated by a structured nonoperative management protocol: an orthosis and exercise program. Foot Ankle Int 2006;27:2-8.

21. Augustin JF, Lin SS, Berberian WS, et al. Nonoperative treatment of adult acquired flat foot with the Arizona brace. Foot Ankle Clin 2003;8:491-502.

22. Garber CE, Blissmer B, Deschenes MR, et al. Quantity and quality of exercise for developing and maintaining cardiorespiratory, musculoskeletal, and neuromotor fitness in apparently healthy adults: guidance for prescribing exercise. Med Sci Sports Exerc 2011:43:1334-59.

23. Moher D, Liberati A, Tetzlaff J, et al. Preferred reporting items for systematic reviews and meta-analyses: the PRISMA statement. BMJ 2009;339:b2535.
24. Higgins PTJ, Green S. Cochrane handbook for systematic reviews of interventions. West Sussex: Sons Ltd, 2008.

25. Ross MH, Smith MD, Vicenzino B. Reported selection criteria for adult acquired flatfoot deformity and posterior tibial tendon dysfunction: are they one and the same? A systematic review. PLoS One 2017;12:e0187201.

26. Hoffmann TC, Glasziou PP, Boutron I, et al. Better reporting of interventions: template for intervention description and replication (TIDieR) checklist and guide. BMJ 2014;348:g1687.

27. Toigo M, Boutellier U. New fundamental resistance exercise determinants of molecular and cellular muscle adaptations. Eur $J$ Appl Physiol 2006:97:643-63.

28. Holden S, Rathleff MS, Jensen MB, et al. How can we implement exercise therapy for patellofemoral pain if we don't know what was prescribed? A systematic review. Br J Sports Med 2018;52:385.

29. Hopkins WG. 2007. A new view of statistics. Internet society for sport science. http://wwwsportsciorg/resource/stats/indexhtml.

30. Landis JR, Koch GG. The measurement of observer agreement for categorical data. Biometrics. 1977;33:159-74.

31. Jeong TH, JK O, Lee HJ. The effect of the combined stretching and strengthening exercise on the clinical symptoms in posterior tibia tendon dysfunction patient. J Korean Foot Ankle Soc 2008;12:47-54

32. Houck J, Neville C, Tome J, et al. Randomized controlled trial comparing orthosis augmented by either stretching or stretching and strengthening for stage II tibialis posterior tendon dysfunction. Foot Ankle Int 2015;36:1006-16.

33. Kulig K, Reischl SF, Pomrantz AB, et al. Nonsurgical management of posterior tibial tendon dysfunction with orthoses and resistive exercise: a randomized controlled trial. Phys Ther 2009;89:26-37.

34. Hunt KJ, Hurwit D. Use of patient-reported outcome measures in foot and ankle research. J Bone Joint Surg Am 2013;95:e118-

35. Cook JL, Docking SI. "Rehabilitation will increase the "capacity" of your ....insert musculoskeletal tissue here...." Defining 'tissue capacity': a core concept for clinicians. Br J Sports Med 2015;49:1484-5.

36. Magnusson SP, Langberg $\mathrm{H}$, Kjaer $\mathrm{M}$. The pathogenesis of tendinopathy: balancing the response to loading. Nat Rev Rheumatol 2010;6:262-8.

37. Cook JL, Rio E, Purdam CR, et al. Revisiting the continuum model of tendon pathology: what is its merit in clinical practice and research? Br J Sports Med 2016;50:1187-91.

38. Kingma JJ, de Knikker R, Wittink HM, et al. Eccentric overload training in patients with chronic Achilles tendinopathy: a systematic review. Br J Sports Med 2007;41:e3.

39. Magnussen RA, Dunn WR, Thomson AB. Nonoperative treatment of midportion Achilles tendinopathy: a systematic review. Clin J Sport Med 2009;19:54-64.

40. Malliaras P, Maffulli N, Garau G. Eccentric training programmes in the management of lateral elbow tendinopathy. Disabil Rehabil 2008;30(20-22):1590-6.

41. Visnes $\mathrm{H}$, Bahr R. The evolution of eccentric training as treatment for patellar tendinopathy (jumper's knee): a critical review of exercise programmes. Br J Sports Med 2007;41:217-23.

42. Young MA, Cook JL, Purdam CR, et al. Eccentric decline squat protocol offers superior results at 12 months compared with traditional eccentric protocol for patellar tendinopathy in volleyball players. Br J Sports Med 2005;39:102-5.

43. Mafi N, Lorentzon R, Alfredson $\mathrm{H}$. Superior short-term results with eccentric calf muscle training compared to concentric training in a randomized prospective multicenter study on patients with chronic Achilles tendinosis. Knee Surg Sports Traumatol Arthrosc 2001;9:42-7.

44. Silbernagel KG, Thomeé R, Thomeé P, et al. Eccentric overload training for patients with chronic Achilles tendon pain-a randomised controlled study with reliability testing of the evaluation methods. Scand J Med Sci Sports 2001;11:197-206.

45. Mellor R, Bennell K, Grimaldi A, et al. Education plus exercise versus corticosteroid injection use versus a wait and see approach on global outcome and pain from gluteal tendinopathy: prospective, single blinded, randomised clinical trial. BMJ 2018;361:k1662.

46. Malliaras P, Barton CJ, Reeves ND, et al. Achilles and patellar tendinopathy loading programmes. Sports Med 2013;43:267-86.

48. American College of Sports Medicine. ACSM's exercise testing and prescription. Philadelphia: Lippincott Williams \& Wilkins, 2017.

48. Cook JL, Purdam CR. Is tendon pathology a continuum? A pathology model to explain the clinical presentation of load-induced tendinopathy. Br J Sports Med 2009;43:409-16.

49. Soslowsky LJ, Thomopoulos S, Esmail A, et al. Rotator cuff tendinosis in an animal model: role of extrinsic and overuse factors. Ann Biomed Eng 2002;30:1057-63. 
50. Cook JL, Purdam CR. The challenge of managing tendinopathy in competing athletes. Br J Sports Med 2014;48:506-9.

51. Cook JL, Purdam CR. Rehabilitation of lower limb tendinopathies. Clin Sports Med 2003;22:777-89.

52. Khan K, Cook J. The painful nonruptured tendon: clinical aspects. Clin Sports Med 2003;22:711-25.

53. Kountouris A, Cook J. Rehabilitation of Achilles and patellar tendinopathies. Best Pract Res Clin Rheumatol 2007;21:295-316.

54. Silbernagel KG, Thomeé R, Eriksson BI, et al. Full symptomatic recovery does not ensure full recovery of muscle-tendon function in patients with Achilles tendinopathy. Br J Sports Med 2007:41:276-80

55. Knutzen KM, Price A. Lower extremity static and dynamic relationships with rearfoot motion in gait. J Am Podiatr Med Assoc 1994:84:171-80.

56. Koshino Y, Yamanaka M, Ezawa Y, et al. Coupling motion between rearfoot and hip and knee joints during walking and single-leg landing. J Electromyogr Kinesiol 2017;37:75-83.

57. Souza TR, Pinto RZ, Trede RG, et al. Temporal couplings between rearfoot-shank complex and hip joint during walking. Clin Biomech 2010;25:745-8.

58. Tiberio $D$. The effect of excessive subtalar joint pronation on patellofemoral mechanics: a theoretical model. J Orthop Sports Phys Ther 1987;9:160-5.

59. Tiberio D. Pathomechanics of structural foot deformities. Phys Ther 1988:68:1840-9.

60. Powers $\mathrm{CM}$. The influence of altered lower-extremity kinematics on patellofemoral joint dysfunction: a theoretical perspective. J Orthop Sports Phys Ther 2003;33:639-46.
61. Dierks TA, Manal KT, Hamill J, et al. Proximal and distal influences on hip and knee kinematics in runners with patellofemoral pain during a prolonged run. $J$ Orthop Sports Phys Ther 2008;38:448-56.

62. Barwick A, Smith J, Chuter V. The relationship between foot motion and lumbopelvic-hip function: a review of the literature. Foot 2012;22:224-31.

63. Cashman GE. The effect of weak hip abductors or external rotators on knee valgus kinematics in healthy subjects: a systematic review. J Sport Rehabil 2012;21:273-84.

64. Nawoczenski DA, Saltzman CL, Cook TM. The effect of foot structure on the three-dimensional kinematic coupling behavior of the leg and rear foot. Phys Ther 1998;78:404-16.

65. Houck JR, Neville CG, Tome J, et al. Ankle and foot kinematics associated with stage II PTTD during stance. Foot Ankle Int 2009;30:530-9.

66. Neville C, Flemister AS, Houck JR. Deep posterior compartment strength and foot kinematics in subjects with stage II posterior tibial tendon dysfunction. Foot Ankle Int 2010;31:320-8.

67. Rabbito M, Pohl MB, Humble N, et al. Biomechanical and clinical factors related to stage I posterior tibial tendon dysfunction. $J$ Orthop Sports Phys Ther 2011;41:776-84.

68. Tome J, Nawoczenski DA, Flemister A, et al. Comparison of foot kinematics between subjects with posterior tibialis tendon dysfunction and healthy controls. $J$ Orthop Sports Phys Ther 2006;36:635-44.

69. Ness ME, Long J, Marks R, et al. Foot and ankle kinematics in patients with posterior tibial tendon dysfunction. Gait Posture 2008;27:331-9. 